\title{
Imaging by intensity interferometry of x-ray fluorescence at a compact $x$-ray free-electron laser
}

\author{
Andrew S. H. Shevchuk $\odot,{ }^{*}$ John C. H. Spence $\odot$, Richard A. Kirian $\odot$, William S. Graves $\odot$, and Kevin E. Schmidt $\odot$ \\ Department of Physics, Arizona State University, Tempe, Arizona 85287, USA
}

(Received 5 December 2020; revised 13 March 2021; accepted 16 April 2021; published 19 August 2021)

\begin{abstract}
A semiclassical theory of incoherent diffractive imaging is given, based on the Hanbury Brown and Twiss effect when used to image inner-shell x-ray fluorescence from heavy atoms excited by the femtosecond pulses of an x-ray laser. Interference between emission from different atoms is expected when the pulse duration is shorter than the fluorescent lifetime. Simulations for atoms at the vertices of an icosahedral virus capsid are given, and reconstructions are presented based on phasing of the pair correlation function between photons emitted independently from many different atoms at two different detector pixels. The dependence of the paircorrelation function on the fluorescence lifetime relative to the pulse duration of the $\mathrm{x}$-ray free-electron laser (XFEL) is computed, and a simple expression is obtained for the contrast of incoherent diffractive imaging speckles as a function of the XFEL's flux and lifetime. This indicates that compact XFELs, with reduced flux but sub-femtosecond pulses, should be ideally suited to atomic-resolution three-dimensional mapping of heavy atoms in materials science, chemistry, and biology.
\end{abstract}

DOI: 10.1103/PhysRevA.104.023514

\section{INTRODUCTION}

As a result of their war-time experience with radar and the early development of radio astronomy, Hanbury Brown and Twiss (HBT) established (amongst much controversy) that there exists a correlation in the intensity fluctuations of radiation from a partially coherent source, detected at two different places. Since the degree of coherence also depends on source size, this "intensity interferometry" at first provided a method of measuring the angular diameter of the sun and of some radio stars. The first test of the idea using light appeared later in 1956 [1]. The correlation function is formed from a time average of the intensities at two different points after forming their product and is proportional to the second-order degree of coherence. This gives the probability of detecting a photon at one detector pixel given that one has been detected at another. From this, the modulus of the first-order degree of coherence may be obtained. Quantum treatments of this problem lead to the birth of quantum optics [2]. (For a review, see Ref. [3].)

Their result may be applied to a continuous distribution of sources and so can be used to reconstruct an image of the source from intensity correlations in the emitted light. In the two-dimensional case, the complex first-order degree of coherence is related by a Fourier transform to the source intensity distribution (van Cittert-Zernike theorem). Imaging using the HBT effect in which intensity correlations are mea-

\footnotetext{
*andrew.shevchuk@asu.edu
}

Published by the American Physical Society under the terms of the Creative Commons Attribution 4.0 International license. Further distribution of this work must maintain attribution to the author(s) and the published article's title, journal citation, and DOI. sured then requires a solution to the phase problem to obtain the complex degree of coherence. This method of imaging an incoherent (self-luminous) object has been analyzed and demonstrated by several groups to provide super-resolution, in some cases using triple correlations to solve the phase problem [4-6].

It has recently been proposed [7] that this method (known as incoherent diffractive imaging or IDI) might be applied to the inner-shell characteristic emission from atoms in a sample excited by an x-ray free-electron laser (XFEL). HBT imaging using vacuum ultraviolet (vuv) XFEL radiation with a masklike object and diffuser (to degrade coherence) has been demonstrated [8], and the coherence properties of a hard $\mathrm{x}$-ray XFEL have been studied using HBT intensity interferometry [9]. For the simple case of two emitting atoms, their $\mathrm{x}$-ray fluorescence will then be coherent within the emission lifetime (coherence time) if the XFEL pulse is briefer than this lifetime. (The situation is akin to interference effects observed between two different lasers when the detection time is shorter than the reciprocal of their frequency difference.) $\mathrm{X}$-ray interference fringes, akin to Young's fringes, would then be detected, but would be different for each XFEL shot due to random emission phases at each atom. However a stable pattern appears in the visibility of the intensity correlation function or the second-order coherence function, which is not isotropic.

An x-ray imaging method based on this effect would benefit from the much larger cross section for inner-shell fluorescence than for the elastic scattering used for coherent diffractive imaging (CDI). In addition, fluorescence (normally isotropic) may be detected over a very wide range of angles and so eliminates the "beam-stop" problem in CDI, while also offering the possibility of super-resolution imaging, together with the chemical species selectivity of inner-shell fluorescence. 
In this paper we provide a semiclassical theory and simulations for this scheme, and we examine the use of the unique properties of patterned inverse Compton compact XFEL radiation in this method. Our aim is to calculate the resolution and contrast of reconstructed three-dimensional images of bioparticles, such as viruses, and proteins containing fluorescent heavy atoms, as a function of the ratio of the XFEL pulse duration to the lifetime of the inner-shell fluorescence. We use the method of iterated projections to address the phase problem (see Ref. [10] for a review). Where applicable, our calculations are done in Heaviside-Lorentz units.

\section{A SEMICLASSICAL MODEL FOR IDI}

Fluorescence observed in the far field is dominated by the dipole term in Maxwell's equations. We therefore approximate the emitters in the sample by an array of $n$ pointlike, damped dipole radiators each defined by a time-dependent vector:

$$
\mathbf{p}_{n}(t)=\mathbf{p}_{n_{0}} e^{-\Gamma\left(t-t_{n}\right) / 2} \sin \left(\omega_{0} t+\phi_{n}\right) \Theta\left(t-t_{n}\right) .
$$

Here $\mathbf{p}_{n_{0}}$ is a constant dipole-moment vector that spatially orients the emitter and the fluorescence occurs at time $t_{n}$ with the mean angular frequency $\omega_{0}$ and the initial phase $\phi_{n}$, where the latter is defined such that it absorbs a term of the form $\omega_{0} t_{n}$. The damping coefficient $\Gamma$ is the Einstein coefficient for spontaneous emission and the inverse of the decay time of the excited state. It defines the half width at half maximum of a Lorentzian line profile governed purely by radiative broadening, which we assume to be the dominant effect on the linewidth.

We also assume that the complicated intra-atomic physics between photoionization and fluorescence happens on a timescale much shorter than the fluorescence lifetime and can be safely be ignored. Therefore, $t_{n}$ in our model is also when the atom is photoionized and the step function $\Theta\left(t-t_{n}\right)$ ensures that emitters only contribute postexcitation. The initial parameters $\mathbf{p}_{n_{0}}, t_{n}$, and $\phi_{n}$ are treated as random variables for each value of $n$ as discussed in Secs. II A-II C.

From (1), we define the resulting polarization density $\mathbf{P}_{n}(t)=\mathbf{p}_{n}(t) \delta^{3}\left(\mathbf{r}-\mathbf{r}_{n}\right)$ for the emitter with position $\mathbf{r}_{n}$ so that it contributes an electromagnetic current density:

$$
\begin{aligned}
\mathbf{J}_{n}(\mathbf{r}, t) & =\frac{d \mathbf{P}_{n}(\mathbf{r}, t)}{d t} \\
& =\omega_{0} \mathbf{p}_{n_{0}} e^{-\Gamma\left(t-t_{n}\right) / 2} \cos \left(\omega_{0} t+\phi_{n}\right) \delta^{3}\left(\mathbf{r}-\mathbf{r}_{n}\right) \Theta\left(t-t_{n}\right) .
\end{aligned}
$$

For inner-shell $\mathrm{x}$-ray fluorescence, $\omega_{0} \gg \Gamma$, and we henceforth discard all terms of order $\Gamma / \omega_{0}$ as they arise in our calculations.

Maxwell's equations expressed in potential form, assuming the Lorenz gauge, are

$$
\begin{gathered}
\frac{\partial^{2} \Phi(\mathbf{r}, t)}{c^{2} \partial t^{2}}-\nabla^{2} \Phi(\mathbf{r}, t)=\rho(\mathbf{r}, t), \\
\frac{\partial^{2} \mathbf{A}(\mathbf{r}, t)}{c^{2} \partial t^{2}}-\nabla^{2} \mathbf{A}(\mathbf{r}, t)=\frac{1}{c} \mathbf{J}(\mathbf{r}, t) .
\end{gathered}
$$

Using (2), the general solution for the retarded vector potential generated by the $n$th emitter is

$$
\begin{aligned}
\mathbf{A}_{n}(\mathbf{r}, t)= & \frac{1}{4 \pi c} \int \frac{\mathbf{J}_{n}\left(\mathbf{r}^{\prime}, t-\left|\mathbf{r}-\mathbf{r}^{\prime}\right| / c\right)}{\left|\mathbf{r}-\mathbf{r}^{\prime}\right|} d^{3} \mathbf{r}^{\prime} \\
= & \frac{\omega_{0} \mathbf{p}_{n_{0}}}{4 \pi c\left|\mathbf{r}-\mathbf{r}_{n}\right|} e^{-\Gamma\left(t-t_{n}-\left|\mathbf{r}-\mathbf{r}_{n}\right| / c\right) / 2} \\
& \times \cos \left[\omega_{0}\left(t-t_{n}-\left|\mathbf{r}-\mathbf{r}_{n}\right| / c\right)+\phi_{n}\right] \\
& \times \Theta\left(t-t_{n}-\left|\mathbf{r}-\mathbf{r}_{n}\right| / c\right) .
\end{aligned}
$$

We assume the sample's diameter is much smaller than the distance to the detector and that the sample is near the origin of our coordinate system. The far-field approximation then simplifies the term in the denominator to $\left|\mathbf{r}-\mathbf{r}_{n}\right| \approx|\mathbf{r}|=$ $r$ and the terms in the numerator to $\left|\mathbf{r}-\mathbf{r}_{n}\right| \approx r-\hat{\mathbf{r}} \cdot \mathbf{r}_{n}$. Defining for notational clarity $T_{n}=t_{n}+c^{-1} r-c^{-1} \hat{\mathbf{r}} \cdot \mathbf{r}_{n},(5)$ becomes

$$
\begin{aligned}
\mathbf{A}_{n}(\mathbf{r}, t)= & \frac{\omega_{0} \mathbf{p}_{n_{0}}}{4 \pi c r} e^{-\Gamma\left(t-T_{n}\right) / 2} \\
& \times \cos \left[\omega_{0}\left(t-T_{n}+t_{n}\right)+\phi_{n}\right] \Theta\left(t-T_{n}\right) .
\end{aligned}
$$

From this, the vacuum form of (4) simplifies to $\frac{\partial \mathbf{E}_{n}(\mathbf{r}, t)}{c \partial t}=$ $\nabla \times\left[\nabla \times \mathbf{A}_{n}(\mathbf{r}, t)\right]$. Substituting (6), the electric field of the fluorescence is

$$
\begin{aligned}
\mathbf{E}_{n}(\mathbf{r}, t)= & \frac{\omega_{0}^{2}}{4 \pi c^{2} r}\left[\mathbf{p}_{n_{0}}-\left(\mathbf{p}_{n_{0}} \cdot \hat{\mathbf{r}}\right) \hat{\mathbf{r}}\right] e^{-\Gamma\left(t-T_{n}\right) / 2} \\
& \times \sin \left[\omega_{0}\left(t-T_{n}+t_{n}\right)+\phi_{n}\right] \Theta\left(t-T_{n}\right) .
\end{aligned}
$$

As a consistency check, the vacuum relation $\mathbf{B}_{n}(\mathbf{r}, t)=\hat{\mathbf{r}} \times$ $\mathbf{E}_{n}(\mathbf{r}, t)$ yields the same result as $\mathbf{B}_{n}(\mathbf{r}, t)=\nabla \times \mathbf{A}_{n}(\mathbf{r}, t)$.

The total energy radiated by a single emitter located at $\mathbf{r}_{n}$ and fluorescing at time $t_{n}$ is the integral of the Poynting vector $\mathbf{S}_{n}=\mathbf{E}_{n} \times \mathbf{B}_{n}$ over a closed surface $S$ with normal vector $\hat{\mathbf{n}}$ for all $t>t_{n}$ :

$$
\begin{aligned}
W_{n}= & \int_{T_{n}}^{\infty} \int_{S} c\left[\mathbf{E}_{n}(\mathbf{r}, t) \times \mathbf{B}_{n}(\mathbf{r}, t)\right] \cdot d \hat{\mathbf{n}} d t \\
= & \int_{T_{n}}^{\infty} \int_{S} c\left\{\mathbf{E}_{n}(\mathbf{r}, t) \times\left[\hat{\mathbf{r}} \times \mathbf{E}_{n}(\mathbf{r}, t)\right]\right\} \cdot d \hat{\mathbf{n}} d t \\
= & \int_{T_{n}}^{\infty} \int_{S}\left|\mathbf{E}_{n}(\mathbf{r}, t)\right|^{2} r^{2} c d \Omega d t \\
= & \int_{T_{n}}^{\infty} \int_{S} \frac{\omega_{0}^{4}\left|\mathbf{p}_{n_{0}}\right|^{2}}{32 \pi^{2} c^{3}}\left[\hat{\mathbf{p}}_{n_{0}}^{2}-\left(\hat{\mathbf{p}}_{n_{0}} \cdot \hat{\mathbf{r}}\right)^{2}\right] e^{-\Gamma\left(t-T_{n}\right)} \\
& \times \Theta\left(t-T_{n}\right) d \Omega d t \\
= & \frac{\omega_{0}^{4}\left|\mathbf{p}_{n_{0}}\right|^{2}}{12 \pi c^{3} \Gamma} .
\end{aligned}
$$

The right-hand expression in the second line of (8) is the cycle-averaged intensity, and the integral over the solid angle makes use of the identity $\int(\mathbf{a} \cdot \hat{\mathbf{p}})(\mathbf{b} \cdot \hat{\mathbf{p}}) d \Omega_{\hat{\mathbf{p}}}=4 \pi(\mathbf{a} \cdot \mathbf{b}) / 3$. The magnitude of the dipole moment vector is normalized to

$$
\left|\mathbf{p}_{n_{0}}\right|=\sqrt{\frac{12 \pi \hbar c^{3} \Gamma}{\omega_{0}^{3}}}
$$

by requiring that a single photon has the energy $W_{\gamma}=\hbar \omega_{0}$. 
From (8), it is clear that $T_{n}$ can be interpreted as the time fluorescence from the $n$th emitter takes to reach a point on an integrating detector. The cycle-averaged intensity of an ensemble of $N$ emitters observed at the surface of a detector in the direction $\mathbf{k}=\left(\frac{\omega}{c}\right) \hat{\mathbf{r}}$ is

$$
\begin{aligned}
\bar{I}(\mathbf{k}, t)= & \sum_{m, n}^{N} \overline{\mathbf{E}_{m} \cdot \mathbf{E}_{n}} \\
= & \sum_{m, n}^{N} \frac{3 \hbar \omega_{0} \Gamma}{8 \pi c r^{2}}\left[\hat{\mathbf{p}}_{m_{0}} \cdot \hat{\mathbf{p}}_{n_{0}}-\left(\hat{\mathbf{p}}_{m_{0}} \cdot \hat{\mathbf{k}}\right)\left(\hat{\mathbf{p}}_{n_{0}} \cdot \hat{\mathbf{k}}\right)\right] \\
& \times e^{-\Gamma\left(2 t-T_{m}-T_{n}\right) / 2} \\
& \times e^{i\left[\mathbf{k} \cdot\left(\mathbf{r}_{m}-\mathbf{r}_{n}\right)+\phi_{m}-\phi_{n}\right]} \Theta\left[t-\max \left(T_{m}, T_{n}\right)\right],
\end{aligned}
$$

where the normalization (9) has been included and we have converted to reciprocal space coordinates for use at the detector. The total fluence (energy per pulse per unit area) in the $\mathbf{k}$ direction is then

$$
\begin{aligned}
I(\mathbf{k})= & \int_{\infty}^{\infty} \bar{I}(\mathbf{k}, t) c d t \\
= & \sum_{m, n}^{N} \frac{3 \hbar \omega_{0}}{8 \pi r^{2}}\left[\hat{\mathbf{p}}_{m_{0}} \cdot \hat{\mathbf{p}}_{n_{0}}-\left(\hat{\mathbf{p}}_{m_{0}} \cdot \hat{\mathbf{k}}\right)\left(\hat{\mathbf{p}}_{n_{0}} \cdot \hat{\mathbf{k}}\right)\right] e^{-\Gamma\left|T_{m}-T_{n}\right| / 2} \\
& \times e^{i\left[\mathbf{k} \cdot\left(\mathbf{r}_{m}-\mathbf{r}_{n}\right)+\phi_{m}-\phi_{n}\right]} .
\end{aligned}
$$

This expression measures the integrated intensity at a point on the detector and captures the interference of the electric fields, which only overlap significantly if the waves arrive at the same pixel within the coherence time $\tau_{c}=2 / \Gamma$. As (10) has an exponential falloff in $t$ with a coherence time much shorter than the exposure length, integrating over all future time closely approximates a snapshot in essentially all cases. The appearance of the $\left|T_{m}-T_{n}\right|$ term occurs because the wave fronts can arrive at a point on the detector in either order, but the cross terms in $m$ and $n$ will not contribute to (11) until both wave fronts have arrived at that point. The integration variable can be redefined as $t_{\ell}=t-\max \left(T_{m}, T_{n}\right)$, which is the time of the later arrival, and integrated from zero to infinity.

Unlike coherent diffractive imaging, in an intensity interferometry experiment the observable quantity is the secondorder degree of coherence in the field amplitudes:

$$
\gamma^{(2)}(\mathbf{q})=\sum_{i, j} \gamma^{(2)}\left(\mathbf{k}_{i}, \mathbf{k}_{j}\right)=\sum_{i, j} \frac{\left\langle I\left(\mathbf{k}_{i}\right) I\left(\mathbf{k}_{j}\right)\right\rangle}{\left\langle I\left(\mathbf{k}_{i}\right)\right\rangle\left\langle I\left(\mathbf{k}_{j}\right)\right\rangle},
$$

where the angle brackets represent the average over an ensemble of exposures and $\mathbf{q}$ is the set of all $\mathbf{q}_{i j}=\mathbf{k}_{i}-\mathbf{k}_{j}$ for each pair of detector pixels $(i, j)$. In our dipole model we consider the polarization direction, the phase, and the ionization and emission times as the variables of the ensemble that are randomized from exposure to exposure. Importantly, the random ionization times $t_{n}$, the phases $\phi_{n}$, and the dipole moments $p_{n_{0}}$ for all $N$ emitters are assumed to be uncorrelated with each other and the average product of intensities takes the form

$$
\begin{aligned}
\left\langle I\left(\mathbf{k}_{i}\right) I\left(\mathbf{k}_{j}\right)\right\rangle= & \sum_{m, n, m^{\prime}, n^{\prime}}^{N} \frac{9 \hbar^{2} \omega_{0}^{2}}{64 \pi^{2} r^{4}}\left\langle\left[\hat{\mathbf{p}}_{m_{0}} \cdot \hat{\mathbf{p}}_{n_{0}}-\left(\hat{\mathbf{p}}_{m_{0}} \cdot \hat{\mathbf{k}}_{i}\right)\left(\hat{\mathbf{p}}_{n_{0}} \cdot \hat{\mathbf{k}}_{i}\right)\right]\left[\hat{\mathbf{p}}_{m_{0}^{\prime}} \cdot \hat{\mathbf{p}}_{n_{0}^{\prime}}-\left(\hat{\mathbf{p}}_{m_{0}^{\prime}} \cdot \hat{\mathbf{k}}_{j}\right)\left(\hat{\mathbf{p}}_{n_{0}^{\prime}} \cdot \hat{\mathbf{k}}_{j}\right)\right]\right\rangle_{\Omega} \\
& \times\left\langle e^{-\Gamma\left(\left|T_{m i}-T_{n i}\right|+\left|T_{m^{\prime} j}-T_{n^{\prime}}\right|\right) / 2}\right\rangle_{t}\left\langle e^{i\left[\mathbf{k}_{i} \cdot\left(\mathbf{r}_{m}-\mathbf{r}_{n}\right)+\phi_{m}-\phi_{n}\right]} e^{-i\left[\mathbf{k}_{j^{\prime}} \cdot\left(\mathbf{r}_{m^{\prime}}-\mathbf{r}_{n^{\prime}}\right)+\phi_{m^{\prime}}-\phi_{n^{\prime}}\right]}\right\rangle_{\phi} .
\end{aligned}
$$

The bracket subscripts $\Omega, t$, and $\phi$ indicate that these terms are respectively averaged over the solid angle, the ionization time, and the phase. The quantity $T_{m i}$ is the same shorthand expression defined before (6) but now adapted to the direction of the $i$ th pixel. Additional uncorrelated parameters can be added to the model as factors to average over in this expression, including degrees of freedom for the overall position and orientation of the ensemble of emitters, and for whether or not the emitter relaxes through the desired fluorescence channel. The fact that we are assuming the orientation of the sample to be constant in each shot makes our present treatment best suited to the study of fixed targets, but it also applies to any subset of shots collected from randomly oriented samples that have been indexed to have substantially the same orientation. We shall compute the dipole moment and phase averages presently and defer the computation of the emission time average to Secs. II B and II C.

\section{A. Ensemble averaging}

The behavior of the phase factors in the average is the key to achieving nonzero intensity correlations. In computing the average intensity $\left\langle I\left(\mathbf{k}_{i}\right)\right\rangle$ the phase term

$$
\begin{aligned}
& \sum_{m, n}^{N}\left\langle\left. e^{i\left[\mathbf{k} \cdot\left(\mathbf{r}_{m}-\mathbf{r}_{n}\right)+\phi_{m}-\phi_{n}\right]}\right|_{\phi}\right. \\
& \quad=\sum_{m, n}^{N} \frac{1}{4 \pi^{2}} \int_{0}^{2 \pi} \int_{0}^{2 \pi} e^{i\left[\mathbf{k} \cdot\left(\mathbf{r}_{m}-\mathbf{r}_{n}\right)+\phi_{m}-\phi_{n}\right]} d \phi_{m} d \phi_{n}
\end{aligned}
$$

vanishes for all $m \neq n$, and the average intensity simplifies to

$$
\left\langle I\left(\mathbf{k}_{i}\right)\right\rangle=\frac{3 N \hbar \omega_{0}}{8 \pi r^{2}}\left\langle\hat{\mathbf{p}}_{0}^{2}-\left(\hat{\mathbf{p}}_{0} \cdot \hat{\mathbf{k}}_{i}\right)^{2}\right\rangle_{\Omega}=\frac{N \hbar \omega_{0}}{4 \pi r^{2}},
$$

where the integral over the solid angle is the normalized version of the one evaluated in (8). However, the phase term in (13) is

$$
\begin{aligned}
& \sum_{m, n, m^{\prime}, n^{\prime}}^{N}\left\langle e^{i\left[\mathbf{k}_{i} \cdot\left(\mathbf{r}_{m}-\mathbf{r}_{n}\right)+\phi_{m^{\prime}}-\phi_{n}\right]} e^{-i\left[\mathbf{k}_{j} \cdot\left(\mathbf{r}_{m^{\prime}}-\mathbf{r}_{n^{\prime}}\right)+\phi_{m^{\prime}}-\phi_{n^{\prime}}\right]}\right\rangle_{\phi} \\
& =\sum_{m, n, m^{\prime}, n^{\prime}}^{N} \frac{1}{16 \pi^{4}} \int_{0}^{2 \pi} \int_{0}^{2 \pi} \int_{0}^{2 \pi} \int_{0}^{2 \pi} e^{i\left[\mathbf{k}_{i^{\prime}} \cdot\left(\mathbf{r}_{m}-\mathbf{r}_{n}\right)-\mathbf{k}_{j} \cdot\left(\mathbf{r}_{m^{\prime}}-\mathbf{r}_{n^{\prime}}\right)\right]} \\
& \quad \times e^{i\left(\phi_{m}-\phi_{n}-\phi_{m^{\prime}}+\phi_{n^{\prime}}\right)} d \phi_{m} d \phi_{n} d \phi_{m^{\prime}} d \phi_{n^{\prime}} .
\end{aligned}
$$


Clearly, (16) vanishes unless the phase terms cancel and this only happens in three cases: when $m=n=m^{\prime}=n^{\prime}$, when $m=n$ and $m^{\prime}=n^{\prime}$, but $m \neq m^{\prime}$, and when $m=m^{\prime}$ and $n=n^{\prime}$, but $m \neq n$. Simplifying terms, we have

$$
\begin{aligned}
\frac{\left\langle I\left(\mathbf{k}_{i}\right) I\left(\mathbf{k}_{j}\right)\right\rangle}{\left\langle I\left(\mathbf{k}_{i}\right)\right\rangle\left\langle I\left(\mathbf{k}_{j}\right)\right\rangle}= & \sum_{m}^{N} \frac{9}{4 N^{2}}\left\langle\left[1-\left(\hat{\mathbf{p}}_{m_{0}} \cdot \hat{\mathbf{k}}_{i}\right)^{2}\right]^{2}\right\rangle_{\Omega}+\sum_{m \neq m^{\prime}}^{N} \frac{9}{4 N^{2}}\left\langle\left[1-\left(\hat{\mathbf{p}}_{m_{0}} \cdot \hat{\mathbf{k}}_{i}\right)^{2}\right]\left[1-\left(\hat{\mathbf{p}}_{m_{0}^{\prime}} \cdot \hat{\mathbf{k}}_{j}\right)^{2}\right]\right\rangle_{\Omega} \\
& +\sum_{m \neq n}^{N} \frac{9}{4 N^{2}}\left\langle\left[\hat{\mathbf{p}}_{m_{0}} \cdot \hat{\mathbf{p}}_{n_{0}}-\left(\hat{\mathbf{p}}_{m_{0}} \cdot \hat{\mathbf{k}}_{i}\right)\left(\hat{\mathbf{p}}_{n_{0}} \cdot \hat{\mathbf{k}}_{i}\right)\right]\left[\hat{\mathbf{p}}_{m_{0}} \cdot \hat{\mathbf{p}}_{n_{0}}-\left(\hat{\mathbf{p}}_{m_{0}} \cdot \hat{\mathbf{k}}_{j}\right)\left(\hat{\mathbf{p}}_{n_{0}} \cdot \hat{\mathbf{k}}_{j}\right)\right]\right\rangle_{\Omega} \\
& \times\left\langle e^{-\Gamma\left(\left|T_{m i}-T_{n i}\right|+\left|T_{m j}-T_{n j}\right|\right) / 2}\right\rangle_{t} e^{i \mathbf{q}_{i j} \cdot\left(\mathbf{r}_{m}-\mathbf{r}_{n}\right)} .
\end{aligned}
$$

The phase averaging expresses the ergodic principle. The photon phases are taken to be constant once they are generated during a given exposure. However, they are randomized for each subsequent exposure and can be considered fixed on a timescale longer than the exposure time, but shorter than the time between exposures. The limit of an average over infinitely many exposures then yields the same result as averaging the phases over an infinitely long time.

We can make the same argument for the polarization of the emitters. After further averaging the dipole moments over the angle and making use of the angular integral identity before (9) as well as the identity

$$
\begin{aligned}
& \int(\mathbf{a} \cdot \hat{\mathbf{p}})(\mathbf{b} \cdot \hat{\mathbf{p}})(\mathbf{c} \cdot \hat{\mathbf{p}})(\mathbf{d} \cdot \hat{\mathbf{p}}) d \Omega_{\hat{\mathbf{p}}} \\
& \quad=\frac{4 \pi}{15}[(\mathbf{a} \cdot \mathbf{b})(\mathbf{c} \cdot \mathbf{d})+(\mathbf{a} \cdot \mathbf{c})(\mathbf{b} \cdot \mathbf{d})+(\mathbf{a} \cdot \mathbf{d})(\mathbf{b} \cdot \mathbf{c})]
\end{aligned}
$$

we have

$$
\begin{aligned}
& \frac{\left\langle I\left(\mathbf{k}_{i}\right) I\left(\mathbf{k}_{j}\right)\right\rangle}{\left\langle I\left(\mathbf{k}_{i}\right)\right\rangle\left\langle I\left(\mathbf{k}_{j}\right)\right\rangle} \\
& \quad=\frac{9}{10 N}\left[1+\frac{1}{3}\left(\hat{\mathbf{k}}_{i} \cdot \hat{\mathbf{k}}_{j}\right)^{2}\right]+1-\frac{1}{N} \\
& \quad+\sum_{m \neq n}^{N} \frac{1+\left(\hat{\mathbf{k}}_{i} \cdot \hat{\mathbf{k}}_{j}\right)^{2}}{4 N^{2}}\left\langle e^{-\Gamma\left(\left|T_{m i}-T_{n i}\right|+\left|T_{m j}-T_{n j}\right|\right) / 2}\right\rangle_{t} e^{i \mathbf{q}_{i j} \cdot\left(\mathbf{r}_{m}-\mathbf{r}_{n}\right)} .
\end{aligned}
$$

Finally, we can write the last term as a double sum with the $m=n$ contribution subtracted off. Simplifying yields

$$
\begin{aligned}
& \frac{\left\langle I\left(\mathbf{k}_{i}\right) I\left(\mathbf{k}_{j}\right)\right\rangle}{\left\langle I\left(\mathbf{k}_{i}\right)\right\rangle\left\langle I\left(\mathbf{k}_{j}\right)\right\rangle} \\
& =1-\frac{7}{20 N}\left[1-\frac{1}{7}\left(\hat{\mathbf{k}}_{i} \cdot \hat{\mathbf{k}}_{j}\right)^{2}\right] \\
& \quad+\sum_{m, n}^{N} \frac{1+\left(\hat{\mathbf{k}}_{i} \cdot \hat{\mathbf{k}}_{j}\right)^{2}}{4 N^{2}}\left\langle e^{-\Gamma\left(\left|T_{m i}-T_{n i}\right|+\left|T_{m j}-T_{n j}\right|\right) / 2}\right\rangle_{t} e^{i \mathbf{q}_{i j} \cdot\left(\mathbf{r}_{m}-\mathbf{r}_{n}\right)} .
\end{aligned}
$$

The last two terms of (19) make up the normalized correlation of the intensity fluctuations at two pixels on the detector, $\left\langle\Delta I\left(\mathbf{k}_{i}\right) \Delta I\left(\mathbf{k}_{j}\right)\right\rangle /\left\langle I\left(\mathbf{k}_{i}\right)\right\rangle\left\langle I\left(\mathbf{k}_{j}\right)\right\rangle$, which in a traditional HBT experiment contains information about the geometry of the source. Indeed, to the extent that the term averaging over the ionization times is effectively independent of the emitter positions (which are analyzed in Sec. II B), we observe that the double sum factors to $\left|\sum_{m}^{N} e^{i \mathbf{q} \cdot \mathbf{r}_{m}}\right|^{2}=|S(\mathbf{q})|^{2}$, the square of the magnitude of the structure factor familiar from crystallography. From $|S(\mathbf{q})|^{2}$, the real-space charge density can be reconstructed after solving the phase problem. This may be done using iterative methods to obtain the complex structure factors [11]. We note, however, that the vectors $\mathbf{q}_{i j}$ have a different interpretation from conventional diffraction experiments such as CDI or small-angle $\mathrm{x}$-ray scattering-they are defined here for every pair of pixels and not relative to the incident direction of the radiation as they are in the case of elastic scattering. Each shot spans a set $\mathbf{q}$ of vectors $\mathbf{q}_{i j}$ that fill in a three-dimensional (3D) volume rather than a two-dimensional Ewald sphere, and the largest $\left|\mathbf{q}_{i j}\right|$ extends to a resolution higher than that of a typical coherent diffraction pattern. In the special case of only two emitters, $|S(\mathbf{q})|^{2}$ simplifies to $2+$ $2 \cos \left[\mathbf{q} \cdot\left(\mathbf{r}_{2}-\mathbf{r}_{1}\right)\right]=4 \cos ^{2}\left[\mathbf{q} \cdot\left(\mathbf{r}_{2}-\mathbf{r}_{1}\right) / 2\right]$ and the brief interference has the form of the Young's fringes generated by two coherent point sources. Figure 1 presents a standard picture of how interference arises in this simplified case.

We note that our results are similar to the simplified quantum formulation of Ref. [7] for single-photon emitters in that we recover a unit term, a term $O(1 / N)$ to be subtracted, and a term dependent on q. However, (19) includes the contribution of the $m=n=m^{\prime}=n^{\prime}$ term that is absent in their expression. Physically, this term describes the contribution of two photons from the same atom to the pair correlation and should be excluded when considering data generated by single-photon emitters. Subtracting off the contribution of this term in our model would modify the second term in (19) to be $-\frac{5}{4 N}\left[1+\frac{1}{5}\left(\hat{\mathbf{k}}_{i} \cdot \hat{\mathbf{k}}_{j}\right)^{2}\right]$. The intensities in our semiclassical formalism are generated by spherical wave packets and therefore each emitter effectively contributes signal to every pixel, as though it were simultaneously emitting many identical photons. As such the $m=n=m^{\prime}=n^{\prime}$ term contributes in our model and in the simulations of Sec. III based on it, but not to the photon correlations measured in an IDI experiment based on inner-shell fluorescence generated by an XFEL pulse.

\section{B. Experimental geometries for IDI}

We now focus on computing the average over the initial ionization and emission times in the last term of (19),

$$
\mathcal{V}=\left\langle e^{-\Gamma\left(\left|T_{m i}-T_{n i}\right|+\left|T_{m j}-T_{n j}\right|\right) / 2}\right\rangle_{t_{m}, t_{n}},
$$




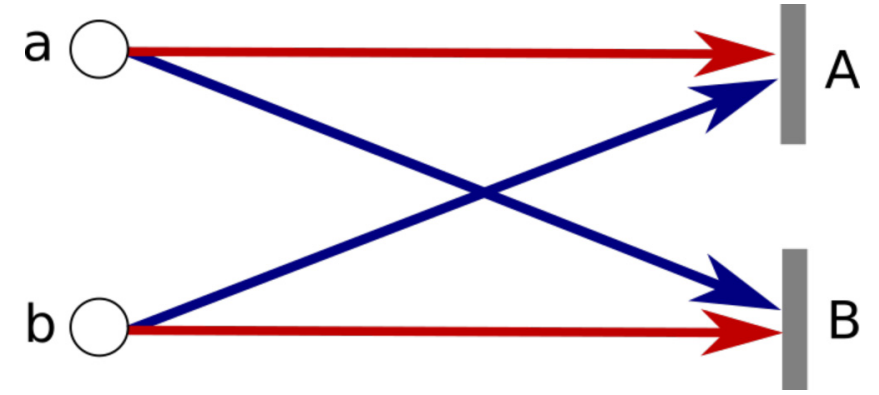

FIG. 1. How can the measurements of two photons be correlated if they are both emitted and detected independently of each other? The reason is that the uncertainty principle forces the observer to trade resolution in time for resolution in energy. If photons emitted at "a" and "b" with frequency difference $\Delta \omega$ (in our case the fluorescence linewidth) have optical path differences to each of detectors " $\mathrm{A}$ " and "B" within a time $\Delta \tau_{c}$ of each other (the fluorescence coherence time), the observer cannot distinguish the photons when $\Delta \omega \Delta \tau_{c}<1 / 2$. Consequently, the two photons could have taken either set of colored paths in the image to the left to arrive at the detectors, and so they are coherent and briefly produce interference fringes. Each path arriving at a detector considered in isolation only contributes to the homogeneous background (since this definitively selects whether the red (top and bottom arrows) or the blue (crossed arrows) paths were followed). Only when the paths are considered as pairs, meaning the joint probability of detecting the photons is considered, does the interference emerge, and with it the ability to extract structural information from the coherence function. We note that this picture only makes sense in the far-field limit, where the spatial modes are indistinguishable.

where we have used the symbol $\mathcal{V}$ because this term functions as the interferometric visibility of the correlation between two pixels. The terms in the exponent of (20) are the time differences for the wave fronts emitted at times $t_{m}$ and $t_{n}$ from emitters located at $\mathbf{r}_{m}$ and $\mathbf{r}_{n}$ to respectively reach pixels $i$ and $j$. We can effectively think of $\mathcal{V}$ as containing all the information about the temporal coherence of the fluorescence, whereas the $e^{i \mathbf{q}_{i j} \cdot\left(\mathbf{r}_{m}-\mathbf{r}_{n}\right)}$ term it multiplies just contains information about the spatial coherence of the fluorescence across the detector.

The importance of $\mathcal{V}$ is that it is a figure of merit for an IDI experiment, so we devote the remainder of Sec. II to studying how to maximize it. This will provide insight on how to optimize the design of future experiments.

First we examine the "ideal" case of a plane-wave pulse that instantaneously ionizes an atom as it sweeps across the sample. The time difference between two emissions for such a pulse is just the projection of the atomic separation along the beam direction (taken to be the $z$ axis): $t_{m}-t_{n}=\hat{\mathbf{z}} \cdot\left(\mathbf{r}_{m}-\right.$ $\left.\mathbf{r}_{n}\right) / c$. Then the "ideal" visibility simplifies to

$$
\mathcal{V}=e^{-\Gamma\left[\left|\mathbf{q}_{i} \cdot\left(\mathbf{r}_{m}-\mathbf{r}_{n}\right)\right|+\left|\mathbf{q}_{j} \cdot\left(\mathbf{r}_{m}-\mathbf{r}_{n}\right)\right|\right] /\left(2 \omega_{0}\right)},
$$

where we have defined $\mathbf{q}_{i}=\omega_{0}\left(\hat{\mathbf{k}}_{i}-\hat{\mathbf{z}}\right) / c$. The reader should note the vectors $\mathbf{q}_{i}$ are precisely those familiar from elastic scattering. They are measured relative to the incident radiation and are fixed to be on the Ewald sphere. The relation between the IDI vectors $\mathbf{q}_{i j}$ and the elastic scattering vectors $\mathbf{q}_{i}$ is just $\mathbf{q}_{i j}=\mathbf{q}_{i}-\mathbf{q}_{j}$.
We can now identify two important and experimentally useful limiting cases that maximize (21):

(i) choose a small sample so that $\mathbf{r}_{m}-\mathbf{r}_{n} \rightarrow 0$,

(ii) place detectors toward the forward direction so that $\mathbf{q}_{i}, \mathbf{q}_{j} \rightarrow 0$.

While the first criterion is somewhat obvious and includes many interesting samples (since the coherence length $c \tau_{c}$ for the fluorescence is optimally of order $100 \mathrm{~nm}$ ), only by placing the detector in the forward direction can we generally maximize $\mathcal{V}$ and observe correlations between the fluorescence emitted at separations much greater than $c \tau_{c}$. This result is nontrivial and indicates that while the fluorescence itself is isotropic, the intensity correlations in the fluorescence are not. Instead, they are strongest in the same direction as the elastic scattering.

Figure 2 demonstrates (21) visually and how the magnitude of the path length difference varies under these ideal pulse conditions for a few simple geometries. Due to the exponential dependence in (21), small changes in the relative path length $c \tau$ due to changes in geometry produce a large change in the signal when the path differences are comparable to the coherence length of the fluorescence. Detecting fluorescence in the direction along the beam has a clear advantage. In effect, forward-directed fluorescence is chasing the x-ray pulse as it sweeps through the sample and so will easily interfere with other forward-directed fluorescence produced at earlier and later times. This makes the coherent volume of the sample very large. Consequently, an IDI experiment at an XFEL looking to image samples larger than the coherence length of the fluorescence should cover as large a solid angle as possible in the forward direction and must filter the elastic scattering. Since the elastic scattering itself is useful for determining which shots are hits and in indexing patterns to find the sample orientation, a geometry in which the fluorescence is detected separately at a low angle off the beam axis is most practical with current detectors.

Furthermore, we see crucially that $\mathcal{V}$ is not independent of the emitter positions, so we cannot simply factor it out of the double sum over emitters to isolate the square of the structure factor and reconstruct the sample geometry. However, we have already established that $\Gamma / \omega_{0}$ is quite small for the lines we are interested in, and if we consider experiments that implement one of the above criteria to maximize $\mathcal{V}$, then to zeroth order we have $\mathcal{V} \approx 1$.

\section{IDI at a compact XFEL}

The previous analysis showed how to optimize the IDI experimental geometry assuming an ideal plane-wave x-ray pulse where ionization occurs instantaneously. In practice, X-ray pulses have a finite duration with some probability to ionize atoms over the length of the pulse and this also affects the visibility. In this section we show that $\mathcal{V}$ as derived in (21) is supplemented by an additional factor accounting for the pulse envelope.

Assume for simplicity a monochromatic, plane-wave $\mathrm{x}$-ray pulse with a time-dependent intensity $I_{0}(t)$ (in photons per unit area per unit time) is incident on a sample. The photoionization rate of an atom in the sample,

$$
\Gamma_{0}(t)=I_{0}(t) \sigma
$$




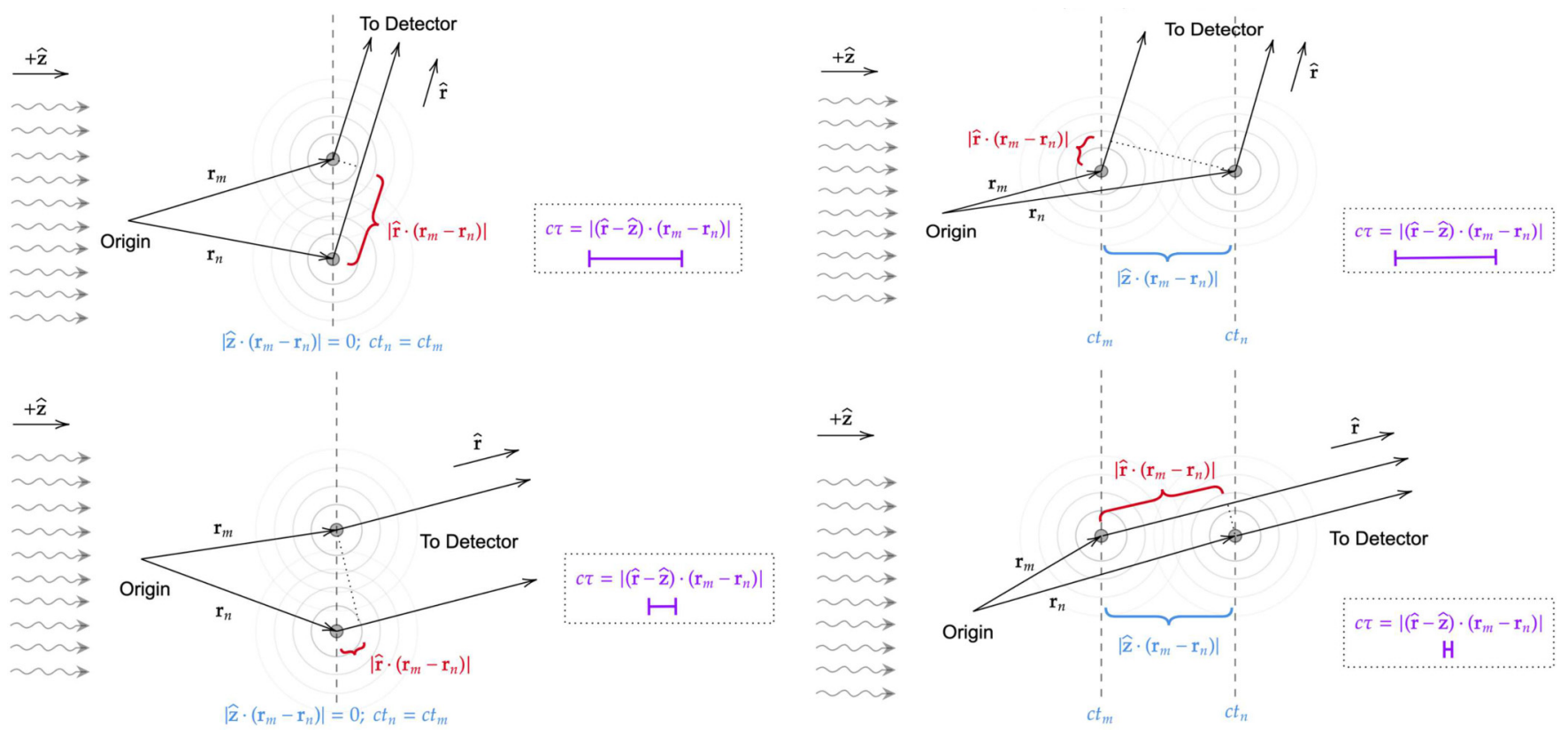

FIG. 2. Four different experimental geometries for IDI are shown for a pair of fluorescence emitters, as are the respective path length differences $c \tau$ (shown by the length of the purple bar) that a photon pair's wave functions are delayed when arriving at one of the pixels on a detector in the far field. This length is the magnitude of the difference in the lengths of the blue and red quantities. In each case the x-ray pulse is taken to originate from the left and is instantaneously ionizing. Notice the distinction in $c \tau$ between the detectors placed way off-axis (top) and those placed slightly off-axis (bottom). Because of the exponential dependence of the visibility on $c \tau$, even a small change in this quantity due to a variation of the geometry can produce a dramatic change in the signal when it is comparable to the coherence length of the fluorescence. Detecting fluorescence in the direction along the beam has a clear advantage over that transverse to the beam.

is determined by the pulse intensity and the atomic photoionization cross section $\sigma$ at the energy of the x-ray photons. We assume that atoms are ionized at most once over the duration of the x-ray pulse. The probability $P_{n}(t+\Delta t)$ that the pulse has ionized the $n$th atom by a time $t+\Delta t$ for some small interval $\Delta t$ is

$$
P_{n}(t+\Delta t)=P_{n}(t)+\left[1-P_{n}(t)\right] \Gamma_{0}(t) \Delta t .
$$

The first term is the the probability that the $n$th atom has been ionized by time $t$ and the second term is the probability that it has not been ionized by time $t$, but is ionized in the small subsequent interval $\Delta t$ with the ionization probability density given by the rate $\Gamma_{0}(t)$. Rearranging to form a difference quotient and taking the limit as $\Delta t \rightarrow 0$ yields a differential equation for the ionization probability:

$$
\frac{d P_{n}(t)}{d t}=\Gamma_{0}(t)\left[1-P_{n}(t)\right] .
$$

$P_{n}(t)$ is interpretable as the cumulative distribution function of possible ionization times for the $n$th atom. Its general solution is

$$
P_{n}(t)=1-e^{\int_{0}^{t} \Gamma_{0}\left(t^{\prime}\right) d t^{\prime}} .
$$

In the simple case of a rectangular x-ray pulse of length $T$ and constant intensity $I_{0}(t)=I_{0}$, we have $P_{n}(t)=1-e^{-\Gamma_{0} t}$. The ionization time of the $n$th emitter is given by $t_{n}=t_{0}+t$, where $t_{0}$ is the time the pulse reaches the $n$th atom and $t_{n}<T$. While an atom will fluoresce some time after $t_{n}$, we take the timescale that the ion lives in an excited state to be negligible, so that $t_{n}$ is also the time at which the $n$th atom emits a photon in our model. This simplification directly links the temporal coherence of the fluorescence to the properties of the XFEL beam.

Considering a plane-wave pulse of constant intensity $I_{0}$ and duration $T$, it is possible to show that the average over $t_{m}$ and $t_{n}$ yields

$$
\mathcal{V}=\left(\frac{U\left(1-e^{-(U+V)}\right)}{(U+V)\left(1-e^{-U}\right)}\right)^{2} e^{-\Gamma\left[\left|\mathbf{q}_{i} \cdot\left(\mathbf{r}_{m}-\mathbf{r}_{n}\right)\right|+\left|\mathbf{q}_{j} \cdot\left(\mathbf{r}_{m}-\mathbf{r}_{n}\right)\right|\right] /\left(2 \omega_{0}\right)} .
$$

Here $U=\Gamma_{0} T$ and $V=\Gamma T$ are two dimensionless parameters relating the characteristic timescales in the system. The interpretation of $U$ is that it is the average number of photons absorbed per emitter per pulse (under the assumption that the emitter could continually absorb $\mathrm{x}$ rays without exhausting its inner-shell electrons). From this model we can directly estimate the overall amplitude of the expected visibility in an IDI experiment. As described in the previous section, if the sample is substantially smaller than $c \tau_{c}$ or the detector is placed in the forward direction, the geometric factor (the exponential term) in (26) is close to unity. Then the magnitude of the visibility is controlled purely by the values of $U$ and $V$.

For clarity, Fig. 3 plots the $U$ and $V$ dependencies in (26). The key relationship is that even a reduction of many orders of magnitude in pulse intensity over that available at current XFELs (the limit of small $U$ ) has very little adverse impact on the visibility so long as the pulse duration is of the order of the coherence time or briefer ( $V$ is of the order of unity or less). To put it another way, we can trade a signal of many weakly correlated photons for one with far fewer strongly correlated photons to achieve the same visibility. 


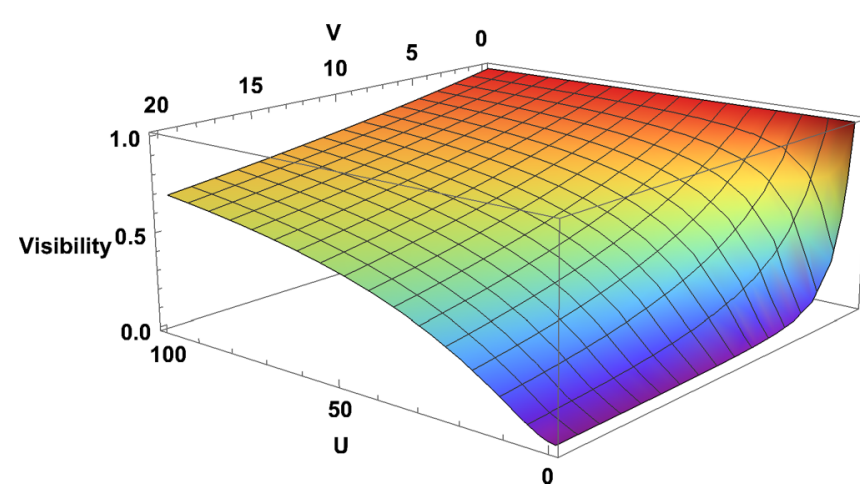

FIG. 3. The prefactor of the visibility function (26) for a rectangular plane-wave pulse is plotted as a function of the ratio of the duration of the XFEL pulse to the lifetime of the atomic inner-shell emission (labeled $V$ ) and the ratio of pulse duration to the inverse ionization rate (labeled $U$ ). For small $U$, the visibility remains high so long as $V \leqslant 1$. In other words, short pulses produce strong correlations even if they are at low intensity. All axes are dimensionless.

Since sub-femtosecond pulses are planned for compact XFELs [12], these machines should be ideal for IDI of heavy atoms provided that pulse fluences averaging roughly one photon per photoabsorption cross section can be achieved (that is, $U=1$ ). With fluences lower than this, a significant number of atoms will not fluoresce, which will reduce the visibility by a factor proportional to the square of the probability of fluorescent emission. We estimate the flux from the compact XFEL under construction at Arizona State University (CXFEL, [13]) by assuming a peak current of $500 \mathrm{~A}$ for 500 as at an electron beam energy of $50 \mathrm{MeV}$ to yield a total stored energy of $12.5 \mu \mathrm{J}$. The FEL process is approximately $0.1 \%$ efficient, yielding a coherent $\mathrm{x}$-ray output of $12.5 \mathrm{~nJ}$ or $10^{7} 7-\mathrm{keV}$ photons per shot. As an example, $\mathrm{Mn}$ has a K-edge $(6.54 \mathrm{keV})$ photoabsorption cross section of $\sigma=4.1 \times 10^{-12} \mu \mathrm{m}^{2}$, which would require a focus area of approximately $4.1 \times 10^{-5} \mu \mathrm{m}^{2}$ (a diameter of roughly $8 \mathrm{~nm}$ ). A beam focus this narrow can be achieved by using a set of multilayer Laue lenses, as described recently by Ref. [14]. Fluences that achieve $U>1$ increase the likelihood that at least one K-shell ionization occurs, but realistically we do not expect more than one useful photon per emitter for IDI. Therefore, there is a "sweet spot" for an IDI experiment at a compact XFEL that achieves $U \geqslant 1$ and $V \leqslant 1$. At a larger machine like LCLS or EuXFEL, $V \leqslant 1$ is unlikely to be achievable and such experiments will need to compensate with $U \gg 1$.

A natural extension of this model is to consider a series of short rectangular pulses with a fixed spacing. In principle, a series of $N_{p}$ pulses of duration $T$ each separated by a time $T_{s}$ would add both intrapulse and interpulse correlations. The latter could be possible if the pulses are sufficiently close together so that $T_{s}$ is not much larger than $\tau_{c}$. Pulse trains are currently in use at the European XFEL, although the spacing of the pulses there, $222 \mathrm{~ns}$, is too large to be useful for IDI. At CXFEL, where sub-femtosecond pulse durations are planned, such a pulse structure should be feasible [15]. The machine is based on the inverse Compton effect and uses a form of electron beam patterning to define the time structure of the x-ray pulses. Electron diffraction at a membrane imposes a spatial pattern across the beam which becomes its time structure after passing through an emittance exchange device. In this way, the time structure may be customized, and temporal coherence retained between groups of pulses.

Last, we note the consequences of the detailed analysis of Ref. [16] studying the signal-to-noise ratio (SNR) of an IDI experiment. The authors found that pairwise correlations among the random phases contribute to the standard deviation of the correlation function with a dependence on the mean number of emitters (rather than the square root of the mean as in the case of Poisson noise). As such, the SNR of an IDI experiment is dominated by this "phase noise" for larger, more complex samples and plateaus to a maximum value, whereas it is dominated by Poisson noise for smaller numbers of emitters. Consequently, using a high-intensity beam at a large XFEL to excite a large sample is subject to diminishing returns with IDI that are not present in the case of CDI, but a tightly focused, sub-femtosecond beam that produces fewer emitters overall, such as that of a compact XFEL, has more favorable SNR characteristics.

\section{SIMULATIONS}

We define an ensemble of fluorescing zinc atoms at fixed positions, assume at most one excitation per atom, and generate IDI snapshots by sampling randomized phases, dipole moments, and ionization times, assuming an ionization rate fixed by the elemental photoabsorption cross section and beam intensity using the model of Sec. II C. The randomized phases are sampled from a uniform distribution while the dipole moment components are sampled from three Gaussian normal distributions and the ionization times are sampled for the cumulative distribution in (25) and are discarded if they extend beyond the time the x-ray pulse interacts with the sample. The incident energy (used for the ionization cross section) is $9.65 \mathrm{keV}$, the energy of the zinc $\mathrm{K}$ edge, and the fluorescence has energy $8.5 \mathrm{keV}$, the zinc $K \alpha$ line. For our compact XFEL simulation we target a 500 -as pulse duration and adjust the pulse intensity to be $2.1 \times 10^{12}$ photons per fs and $\mu \mathrm{m}^{2}$, which yields a value of $U=3$ for the zinc $\mathrm{K}$ shell. For LCLS we assume pulse durations of $3 \mathrm{fs}$ and a pulse intensity of $8.5 \times 10^{11}$ photons per fs and $\mu \mathrm{m}^{2}$. This is optimistic for the current machine parameters at LCLS absent additional optics, but could be achieved with focusing from multilayer Laue lenses. These two sets of parameters were chosen so that they yield roughly the same visibility on both machines.

We define a spherical detector of 48-by-48 pixels where polar and azimuthal directions on the sphere are partitioned into an equal number of pixels. The solid angle subtended by the pixels determines the separation resolvable from interference fringes across the detector. Since $\gamma^{(2)}(\mathbf{q})$ does not explicitly depend on the detector distance, we leave it arbitrary and fix the pixel solid angle instead of the pixel size. With a spherical detector each snapshot captures the largest possible amount of q-space data to be used for a reconstruction.

Using the formula for (11) and a specification of the emitter positions, we compute the intensity at values located in the center of each pixel on our spherical detector and then add 

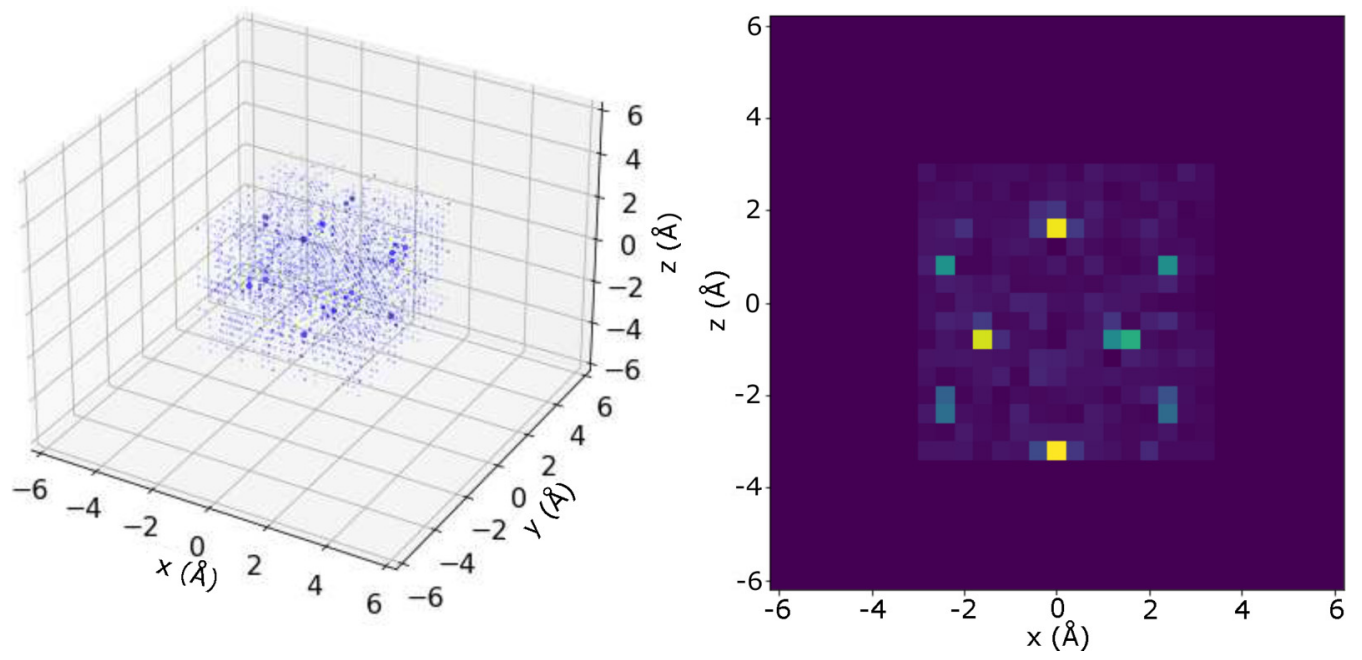

FIG. 4. This figure shows a sample reconstruction of a "virus capsid" of zinc atoms in 3D (left) and projected into the $x$ - $z$, plane (right). The simulation generated and averaged 10000 patterns to compute the degree of coherence assuming emitters from the zinc K shell and instant ionization. Snapshots are simulated as interference patterns on a spherical detector of size $48 \times 48$ pixels and averaged to compute $\gamma^{(2)}(\mathbf{q})$. The $\mathbf{q}$ vectors fill a spherical region in $\mathbf{q}$ space (not shown). A flat detector also provides some 3D $\mathbf{q}$-space data, despite spanning a plane in real space, because the $\mathbf{k}$ vectors to each pixel have constant magnitude while their differences are not restricted to a surface. A cubic support constraint was used for the phasing.

Poisson noise to these values. This is our "snapshot." We then compute $\gamma^{(2)}(\mathbf{q})$ by generating and summing 10000 such interference patterns. In the limit of many patterns, this is the equivalent of averaging over all possible dipole moments, ionization times, and relative phases. After computing the degree of coherence, we bin all $\mathbf{q}$ vectors for every pixel pair into voxels in $\mathbf{q}$ space. Last we implement the hybrid input-output algorithm [11] with a feedback parameter of 0.7 to solve the phase problem and generate a reconstruction.

In Figs. 4 and 5, we have reconstructed a 12-atom icosahedral "virus capsid" of zinc emitters from our simulated low-resolution IDI interference patterns with the abovementioned parameters. The oversampling ratio is roughly a factor of 2 . As noted above, our aim is to show that similar fidelity can be achieved for the different experimental capabilities of a traditional large XFEL and a compact XFEL. While these simulations were done on a laptop computer as a proof of concept and are rather artificial, full-scale IDI simulations could be done in a dedicated high-performance cluster.

\section{DISCUSSION AND CONCLUSIONS}

Given that the experiments of HBT have a well-established methodology whereas x-ray IDI is new, it is useful to outline some of the differences between the two types of experiments. Both experiments measure correlated intensity fluctuations. In an HBT-style experiment, the fluctuations arise as a result of the narrowband frequency filter, which results in a correlation timescale that is inversely proportional to the width of the filter. The output currents of two phototubes are averaged over the response time of the electronics, which is generally longer than the time delay between the photons arriving at the correlator. In an IDI-style experiment the emission signal is gated by the x-ray beam rather than a filter, and exactly when each photon arrives at the detector is unimportant so long as it arrives during the same exposure. This is the value of having an integrating detector over an averaging detector.

As we see from (21) and Fig. 2, the potential to measure correlations over a large distance increases substantially as the detector is placed closer to the forward direction, This is particularly important if the coherence length of the radiation is shorter than ideal due to additional line broadening. In a recent experiment at SACLA [17], nontrivial intensity correlations were observed in $\mathrm{x}$-ray fluorescence with a detector at $30^{\circ}$ off-axis. Their sample was a copper sheet rather than a biomolecule and they were primarily interested in determining the $\mathrm{x}$-ray pulse duration, but this is an important proof of concept that IDI for biomolecular structure determination can be done at XFELs.

Although the derivation of (26) was done using a semiclassical model, we do not believe that a full treatment using quantum electrodynamics would qualitatively change the limiting behavior of the visibility for low-intensity, short-duration pulses that is critical to the success of IDI at a compact XFEL. We compared our results to the simple quantum description given by Ref. [7] of single-photon emitters to show that we recover the same general behavior as they do, but in their model they do not calculate the effects of intensity, pulse duration, and experimental geometry on the visibility of the correlations.

The use of photoelectrons for IDI should also be possible, with the advantages of a direct interaction without "afterglow" and strong signal, but requiring much more complex angle-resolved, time-of-flight photoemission spectroscopy instrumentation. For photoelectrons the coherence time is inversely proportional to the bandwidth of the exciting XFEL beam, since the radiative decay time is negligible for very narrow inner-shell bands from which these photoelectrons originate. The relatively short inelastic mean free path of photoelectrons would restrict the method to smaller molecules, and multiple scattering of the photoelectrons may 



FIG. 5. Three reconstructions and a reference image of a 12-atom icosahedral zinc virus capsid are shown for different experimental IDI parameters. Clockwise from the top left are the reference structure, a reconstruction assuming the ionization occurs instantaneously, an example reconstruction with CXFEL parameters, and an example reconstruction with LCLS parameters. The pulse durations used for LCLS and CXFEL were $3 \mathrm{fs}$ and 500 as, respectively. For instant ionization the pulse is a Dirac $\delta$ function and as expected produces the best reconstruction. The two bottom panels show that similar visibility can be achieved for a reconstruction at either large XFELs or compact XFELs, subject to making the pulse duration as short as possible and focusing the beam with multilayer Laue lenses to achieve higher incident intensities on the sample.

also limit the sample size. Alternatively, multiple photoelectron scattering events could provide additional structural information not present in the photon case. One clear advantage photoelectron IDI would have over x-ray fluorescence is that detection of lighter elements would be possible because the signal is not limited by their low fluorescence yield. To weigh the various pros and cons, an extensive analysis of the feasibility of IDI using photoelectrons instead of x-ray fluorescence should be carried out.

In conclusion, compact XFELs and large XFELs are both capable of performing IDI experiments despite their different scales. We have shown through a semiclassical model of IDI that fluorescence detected in the forward direction provides the strongest intensity correlations. Additionally, we find that the reduced pulse intensity available at compact XFELs can be compensated by the use of the attosecond pulses comparable in duration to the inner-shell fluorescence lifetime of heavy atoms and the use of multilayer Laue lenses to focus the beam onto the sample. With these advantages, IDI is a technique well-suited to this new, smaller class of XFEL.

\section{ACKNOWLEDGMENTS}

This research was funded by the National Science Foundation STC Award No. 1231306 and the National Science Foundation Division of Biological Infrastructure CAREER Award No. 1943448. Any opinions, findings, and conclusions or recommendations expressed in this material are those of the authors and do not necessarily reflect the views of the National Science Foundation.
[1] R. H. Brown and R. Q. Twiss, Correlation between photons in two coherent beams of light, Nature (London) 177, 27 (1956).

[2] Louden, The Quantum Theory of Light (Oxford University, New York, 1973).
[3] R. Brown, The Intensity Interferometer: Its Application to Astronomy (Taylor \& Francis, London, 1974).

[4] G. Shoulga and E. N. Ribak, Toward spectral intensity interferometry, Appl. Opt. 56, A23 (2017). 
[5] A. Classen, F. Waldmann, S. Giebel, R. Schneider, D. Bhatti, T. Mehringer, and J. von Zanthier, Superresolving Imaging of Arbitrary One-Dimensional Arrays of Thermal Light Sources Using Multiphoton Interference, Phys. Rev. Lett. 117, 253601 (2016).

[6] T. Sato, S. Wadaka, J. Yamamoto, and J. Ishii, Imaging system using an intensity triple correlator, Appl. Opt. 17, 2047 (1978).

[7] A. Classen, K. Ayyer, H. N. Chapman, R. Röhlsberger, and J. von Zanthier, Incoherent Diffractive Imaging via Intensity Correlations of Hard X Rays, Phys. Rev. Lett. 119, 053401 (2017).

[8] R. Schneider, T. Mehringer, G. Mercurio, L. Wenthaus, A. Classen, G. Brenner, O. Gorobtsov, A. Benz, D. Bhatti, L. Bocklage et al., Quantum imaging with incoherently scattered light from a free-electron laser, Nat. Phys. 14, 126 (2017).

[9] O. Y. Gorobtsov, N. Mukharamova, S. Lazarev, M. Chollet, D. Zhu, Y. Feng, R. P. Kurta, J.-M. Meijer, G. Williams, M. Sikorski et al., Diffraction based Hanbury Brown and Twiss interferometry at a hard x-ray free-electron laser, Sci. Rep. 8, 1 (2018).

[10] J. C. H. Spence, Diffractive (lensless) imaging, in Science of Microscopy, edited by P. W. Hawkes and J. C. H. Spence (Springer, New York, 2007), pp. 1196-1227.

[11] J. R. Fienup, Phase retrieval algorithms: A comparison, Appl. Opt. 21, 2758 (1982).
[12] E. A. Nanni, W. S. Graves, and D. E. Moncton, Nanomodulated electron beams via electron diffraction and emittance exchange for coherent x-ray generation, Phys. Rev. Accel. Beams 21, 014401 (2018).

[13] W. Graves, J. Chen, P. Fromme, M. Holl, R. Kirian, L. Malin, K. Schmidt, J. Spence, M. Underhill, U. Weierstall et al., ASU compact XFEL, in Proceedings of the 38th International Free Electron Laser Conference (Joint Accelerator Conferences Website (JACoW), Geneva, Switzerland, 2017).

[14] S. Bajt, M. Prasciolu, H. Fleckenstein, M. Domaracký, H. Chapman, A. Morgan, O. Yefanov, M. Messerschmidt, Y. Du, K. Murray, V. Mariani, M. Kuhn, S. Aplin, K. Pande, P. Villanueva-Perez, K. Stachnik, J. Chen, A. Andrejczuk, A. Meents, and C. Hamm, X-ray focusing with efficient high-NA multilayer Laue lenses, Light: Sci. Appl. 7, 17162 (2018).

[15] W. Graves, S. Chang, D. Dwyer, P. Fromme, M. Holl, B. Levin, L. Malin, J. Vincent, J. Spence, E. Nanni et al., Nanopatterned electron beams for temporal coherence and deterministic phase control of x-ray free-electron lasers, arXiv:1906.01525.

[16] F. Trost, K. Ayyer, and H. N. Chapman, Photon statistics and signal to noise ratio for incoherent diffraction imaging, New J. Phys. 22, 083070 (2020).

[17] I. Inoue, K. Tamasaku, T. Osaka, Y. Inubushi, and M. Yabashi, Determination of X-ray pulse duration via intensity correlation measurements of X-ray fluorescence, J. Synchrotron Radiat. 26, 2050 (2019). 